\title{
Study on Dormancy of Words and Causes of Their Activation
}

\author{
Qinggang Ding ${ }^{1}$ \\ ${ }^{1}$ Sichuan University, Chengdu, Sichuan, 610064 \\ ${ }^{1}$ Sichuan University of Arts and Science, Dazhou, Sichuan, 635000
}

Keywords: Words; Dormancy; Manifestation; Motivation

\begin{abstract}
Vocabulary is a collection of all words in a language system. Words that exit the historical stage are not completely "dead", but exist in the form of "dormant". Dormant words have different manifestations in terms of time, scope, and method. Social development, historical culture, and psychological cognition are the main motivations for the activation of dormant words.
\end{abstract}

\section{Introduction}

"Dormancy" is a biological concept, which means that certain living things in extreme environmental conditions, extreme reduction of life activities, into a lethargic state, after the adverse environment has passed, and then re-awakened, growing as usual, activities, such as "plants Dormancy, animal dormancy, etc. With the generalization of the word "dormant", "computer dormancy" and "love dormancy" have emerged. In fact, in the course of development, language also has such a state. We can call it "linguistic dormancy." The vocabulary is most influenced by society, and it is also the fastest in language. Therefore, the most obvious manifestation of language dormancy is word sleep.

\section{The Expression of Dormant Words}

Vocabulary is the most active part of the development of language change. Different parts of the vocabulary system have different characteristics and characteristics, and the situations that they show in the development of change are also different. The following takes the Chinese as an example to analyze three aspects of sleep time, scope, and reasons.

From the perspective of time, it can be divided into short-term sleep words, long-term sleep words and permanent sleep words.

Short sleep words. The major stage of Chinese history is usually a period of 400-500 years. The 500-year period is very long, but it is relatively short-lived compared to the Chinese with a long history. Therefore, we refer to words that are within 500 years of sleep time as short-term sleep words. Such as "fur" word, Yang Lin believes that the "fur" of the "fur skin" meaning is useful in the literature of the Qing Dynasty and is a common word. However, after the 1949 mainland China, especially the Northern Territory, the word "fur" almost disappeared. This may be related to the reform of the political system and ideology. After the reform and opening up, people were emancipated, and they copied Hong Kong's name "fur". This can be said to be a rejuvenation of old words, and it can also be said to be a new word introduced in Hong Kong words [1]. Through Yang Lin's research, we can clearly understand the sleeping time and activation motive of the word "fur". Short-term dormant words are short-lived due to their withdrawal from the historical stage, and many are still stored in people's memory, and are therefore most easily activated.

Long-term dormant words. Long-term dormant words are relative to short-term dormant words, and refer to words with dormant periods of more than 500 years. The words that entered the dormant state, after going through about 500 years, still fail to activate and continue to appear in the vocabulary system, and will enter long-term hibernation. For example, there are a total of 120 horse-related words, which basically have already withdrawn from the stage of history. In general, long-term dormant words are more difficult to activate than short-term dormant words, because these words are no longer exist in some of the things they represent; some things continue to exist, 
but the concepts that represent things have been generalized, such as various types Although horses still exist, due to social development and productivity enhancement, the role of horses in human social life has gradually diminished. Therefore, horse-related words are no longer concerned.

Permanent sleep words. Permanent sleep is another state of long-term hibernation. It mainly means that some words will never be activated in a language system after they sleep. In theory, such words must exist because the language is constantly changing and developing. There are natural laws of metabolism inside the vocabulary system. This is also the need for internal balance of the language system. However, among the specific languages, it is not very clear at the moment what are the words of permanent hibernation. Some words that hibernate with the extinction of old things seem to have become words of permanent dormancy, but they cannot be easily concluded. For example, the names of ancient government officials such as "The prime minister, the minister, the government, the prince, the sealer”, etc., which have not been activated yet, do not mean that these words will not be activated permanently. So the words of permanent dormancy may not be well defined in a living language.

From the perspective of scope, it can be divided into industrial vocabulary hibernation, regional vocabulary hibernation, and colloquial vocabulary hibernation.

Industry vocabulary dormancy. Industry vocabulary sleep can also be called domain vocabulary sleep. Different occupations, groups, organizations, etc. have their own unique vocabulary system. Certain vocabularies in these industries cannot appear in these industries or occupations for various reasons, and they can be called industry dormancy. With the development of the times, industry terms will change. The first is that the rise and fall of the industry determines the increase and decrease of industry vocabulary. When an industry flourishes, the vocabulary will increase dramatically; and when an industry shrinks and disappears, the words of the industry will shrink. The second is that the development of science and technology will cause certain words in the industry to exit the industry's historical stage and become dormant words. Since ancient times, China has been a large agricultural country. Agriculture has a lot of words. In the Ming dynasty Xu Guangqi's Agricultural Encyclopaedia, only a dozen kinds of tools were sown in agricultural implements, such as "cars, cars, gongs, and gongs," because of productivity. To improve, these things have almost disappeared, and the words that refer to these things sleep in the industry vocabulary system.

Regional vocabulary hibernation. The dormancy of regional words is to a large extent the dormancy of dialect words, that is, certain words in a certain dialect area no longer continue to be used on the historical stage. This is mainly related to language contact. There are phenomena of language contact between different languages and different variants within the same language. Influenced by language contact, the exchange and integration between different dialect areas and between dialects and conversational languages will lead some dialect words to gradually withdraw from the use of the local dialect and accept other dialect vocabulary or vocabulary. At the same time, there are also some non-allegative words that have been replaced by other words to enter a dormant state due to avoidance, taboo, etc. in some areas.

Tongue vocabulary sleep. Compared with the regional vocabulary dormancy, the vocabulary dormancy of the common language refers to the fact that certain words commonly used by the people are extremely low in frequency of use or directly quit the speech system. "In the vocabulary, of course, certain words are often used by certain classes and certain groups, but this is only a very small part, and more words have the universality and wideness of the super class and the super group."[2] The development of the vocabulary itself will make many words go to sleep. For example, in the development of Chinese vocabulary from monosyllables to polysyllabic words, many monosyllabic words are gradually replaced by polysyllabic words, such as "eye" instead of "head" and "road" instead of "total". These are replaced Most of the monosyllabic words can only exist as morphemes and are rarely used alone. In addition, the competition between dialects and colloquialism can also lead to the withdrawal of the colloquial vocabulary from the verbal communication system. In general, the general word sleep may start with a small range of industries, regions, etc., and finally affect the use of common words. For example, the fishermen's navigation 
taboos "live", then they describe as "quick" and later write "chopsticks". The dormancy of such words is due to the influence of industrial dormant words on the colloquial expressions.

Natural sleep words. Due to the development of society and the internal competition and adjustment mechanism of the language system, the vitality of certain words gradually weakened. Finally, the language communication system is automatically quitted. Such words can be considered as natural dormancy because of their dormancy. With the demise of old things, many old words automatically exit the stage of history. For example, "Big Brother" is a popular name for mobile phones. It was popular in the 1980s. However, with the continuous improvement of information technology, "Big Brother" was soon replaced by "mobile phones" and "mobile phones." The name of such things naturally disappeared into the speech system. Although something has always existed, the names of things are constantly changing.

Forced to sleep words. Political reforms and language policies in a given period of time can lead to the inability of certain words to appear within the scope of people's use. The dormancy of these words belongs to forced dormancy. At the same time, the "escape" and "taboo" in society can also cause the words to be dormant. The words "straight speak" cannot be forced to suspend use or replace with other words because of avoidance or taboo. For example, in order to avoid the name of Qin Shihuang's ruling politics, people used "end month" instead of "first month", and the word "first month" was forced to become "dormant word." These words cause people to say or not dare to say because of reasons other than the language system. To express these concepts, they must find other words instead. Being forced into dormancy has obvious epochal characteristics. When the reasons for the dormancy of these words are gradually disappeared in the course of social development, these words usually relieve sleep and return to people's use.

\section{The Activation of Sleep Words}

In the whole vocabulary system, why do some sleep words activate and use, and some dormant words are irritated? This mainly depends on the dormant words themselves and their external motivation. The dormant words themselves are also important mechanisms within the language system, and the factors outside the vocabulary system are the key to the activation of dormant words. The activation of dormant words is mainly related to social development, historical culture and psychological cognition. It is under the stimulation of these external motivations that dormant words have the opportunity to activate, and thus enter the language communication system again.

The causes of social development. Social development is a realistic soil for the activation of dormant words. Whether or not dormant words can be activated depends to a large extent on the social reality. The relationship between vocabulary and society is the closest. Many factors such as political changes in social development, economic development, and technological innovation will directly or indirectly affect the use of words. Therefore, many dormant words will be reactivated in the development of society. Social development has prompted the activation of dormant words mainly in three aspects: First, social development has reactivated some common words. Because social development generally takes on a "spiral" rise, many things that have disappeared have reappeared with the development of society. Second, major events or important changes in social development will activate sleep phrases. After the reform and opening up, China's economy has developed rapidly. A large number of dormant terms related to economic activities, such as "pawnshops, exchanges, auctions, opening, stocks, and banknotes" have re-entered the field of vision. Third, social development will influence the change of values, and the words attached to this value will gain the opportunity to be activated. For example, the words "mother, miss, young master, and boss" were used to activate and use because of changes in people's values after the reform and opening up. Therefore, changes in the values of social development also play an important role in the activation of dormant words.

Historical and cultural factors. Language and culture have a close relationship. Language is the mirror image of culture and culture is the foundation of language. The historical documents recorded through language are not only the treasures of ideology and culture, but also the source of constant renewal of vocabulary. Those words that have withdrawn from communication can only be 
preserved in ancient literature, and they will enter the horizons of future generations as future generations study these documents, and may be accompanied by later references to these documents and public historical events. The review was re-enabled. Of course, languages in different cultural contexts have different attitudes toward the composition of extinction. Advocating tradition and respecting the wisdom of the predecessors will prompt people to seek more appropriate means of expression from previous generations of literature. The different focuses of different eras have led to differences in the interest of future generations in different previous generations of literature and old Japanese terms. The words that are of particular concern to later generations will return to everyday communication. This is one of the important reasons why some dormant words can be activated while others are not.

In addition, the mode of transmission of historical culture is particularly important for the activation of dormant words. In today's advanced modes of communication, some dormant words stored in historical documents can quickly enter people's field of vision through various channels such as newspapers, radio, television, and the Internet. For example, with the hits of TV dramas such as "Tuo Biography" and "Yueyue Biography", many words that have been dormant have become known and accepted by people. Therefore, the fast and diverse communication media plays a role in activating the dormant words. Of course, not all dormant words can be activated in the process of historical and cultural communication. Some words can be accepted by people and re-enter the communication system, while some words can only be short-lived.

Psychological cognitive motivation. The activation of dormant words is not only influenced by social development and historical and cultural factors, but also has a great relationship with pragmatic psychological and cognitive mechanisms. "A lot of times, changes in the external world, including natural or social changes, are the main drivers of language change, but the effects of subjective actions on language change are also obvious." [3] The subjective role here is to activate dormant words. It is mainly reflected in the psychological cognition of the choice and acceptance of words. It is precisely under the influence of pragmatic psychology such as novelty, difference, curiosity, and elegance that people often use the words "not to go the unusual way", like to create new words or directly enable dormant words. Of course, the dormant words that are used under the influence of pragmatic psychology can be accepted by people and further developed into official words in communication, and it remains to be tested.

The cognitive mechanism of metaphor and metonymy. Metaphor and metonymy are important tools for human cognitive language and therefore play an important role in the activation of dormant words. In the article Metaphor, Metonymy, Lu Yuming argues that metaphor and metonymy do not "project from one cognitive domain to another cognitive domain," but "activate one cognitive domain from another cognitive domain", and He further proposed that the single activation mainly includes "similarity activation", "integral-partial association activation," and "cause-effect" reasoning association activation". These are also important ways to activate dormant words. When new things, new phenomena, and new concepts emerge in society, and the current language system lacks corresponding reference symbols, people will use metaphor and metonymy cognitive methods based on semantic similarity and relevance. Find the right expression in the entire language thesaurus. In this situation, many dormant words have returned to the stage of communication.

\section{References}

[1] Yang Lin. Sources of Fur and Cloth[J]. Macao Journal of Linguistics, 2014(1):86-87.

[2] Su Xinchun. The measurement of Chinese vocabulary [M]. Xiamen: Xiamen University Press, 2002: 46.

[3] Yu Liming. Vocabulary refurbishment and motivation [A]// Journal of Chinese History: Fifteenth Series. Shanghai: Shanghai Education Agency, 2015: 120-127.

[4] Lu Shuming. Metaphor, metonymy discussion [J]. Foreign Languages, 2009 (1): 44-50. 